\title{
Non-invasive real-time autonomic function characterization during surgery via continuous Poincaré quantification of heart rate variability
}

\author{
Maddalena Ardissino $^{1}$ (1) $\cdot$ Nicoletta Nicolaou ${ }^{2,3,4} \cdot$ Marcela Vizcaychipi $^{1,5}$
}

Received: 8 May 2018 / Accepted: 27 September 2018 / Published online: 3 October 2018

(c) The Author(s) 2018

\begin{abstract}
Heart rate variability (HRV) provides an excellent proxy for monitoring of autonomic function, but the clinical utility of such characterization has not been investigated. In a clinical setting, the baseline autonomic function can reflect ability to adapt to stressors such as anesthesia. No monitoring tool has yet been developed that is able to track changes in HRV in real time. This study is a proof-of-concept for a non-invasive, real-time monitoring model for autonomic function via continuous Poincaré quantification of HRV dynamics. Anonymized heart rate data of 18 healthy individuals (18-45 years) undergoing minor procedures and 18 healthy controls (21-35 years) were analyzed. Patients underwent propofol and fentanyl anesthesia, and controls were at rest. Continuous heart rate monitoring was carried out from before aesthetic induction to the end of the surgical procedure. HRV components (sympathetic and parasympathetic) were extracted and analyzed using Poincaré quantification, and a real-time assessment tool was developed. In the patient group, a significant decrease in the sympathetic and parasympathetic components of HRV was observed following anesthesia (SD1: $p=0.019 ; \mathrm{SD} 2: \mathrm{p}=0.00027$ ). No corresponding change in HRV was observed in controls. HRV parameters were modelled into a real-time graph. Using the monitoring technique developed, autonomic changes could be successfully visualized in real-time. This could provide the basis for a novel, fast and non-invasive method of autonomic assessment that can be delivered at the point of care.
\end{abstract}

Keywords Intraoperative monitoring $\cdot$ Real-time monitoring $\cdot$ Autonomic function $\cdot$ Poincaré $\cdot$ Heart rate variability

\section{Introduction}

Presented at the Intensive Care Society's State of the Art 2017 meeting, Liverpool, December 2017.

Electronic supplementary material The online version of this article (https://doi.org/10.1007/s10877-018-0206-4) contains supplementary material, which is available to authorized users.

Maddalena Ardissino

ma5713@imperial.ac.uk

1 Imperial College School of Medicine, Imperial College London, London SW7 2AZ, UK

2 University of Nicosia Medical School, 21 Ilia Papakyriakou, Egkomi, 2414 Nicosia, Cyprus

3 Department of Electrical and Electronic Engineering, Imperial College London, London SW7 2AZ, UK

4 Biomedical Engineering, School of Biological Sciences, University of Reading, Reading RG6 6AY, UK

5 Magill Department of Anaesthesia, Intensive Care and Pain Management, Chelsea and Westminster Hospital, 369

Fulham Road, London SW10 9NH, UK
The autonomic nervous system (ANS) consists of two main components, the sympathetic and the parasympathetic nervous system (SNS and PSNS respectively), which are responsible for a wide variety of multisystem homeostatic changes, and play a part in the modulation of heart rate variability (HRV). Heart rate variability (HRV) provides an potential proxy for characterization of autonomic nervous system function. Ultimately, variability in heart rate results from continuous modulation of the sino-atrial node (SAN) by the autonomic nervous system, which varies in response to multiple factors such as respiratory rate [1], homeostatic reflexes and centrally generated physiological patterns. Together, these factors influence the sympathovagal balance; it is this balance that ultimately defines the heart rate variability [2]. In a clinical setting, impairments in autonomic function $[3,4]$ may be reflected by changes in heart rate variability [2]. As autonomic dysregulation is a major risk factor for complications of anesthesia such as 
bradycardia and hypotension [5-8], information regarding the autonomic function of patients both before and during anesthesia can be of great value to anesthetists. Thus far, no routine, point-of-care monitoring system has been developed to assess autonomic function in patients before and during anesthesia. Heart rate variability has been shown to mirror changes in autonomic function $[9,10]$, which can be evaluated through the use of Poincaré plots. Poincaré plots are non-linear, geometrical representations of HRV dynamics over a period of time $[11,12]$, in which the HR value at a given time, $\mathrm{HR}(\mathrm{t})$, is plotted against the value of the next HR value, $\operatorname{HR}(\mathrm{t}+1)$ throughout the duration of the recording (Fig. 1) [12]. Each point therefore represents the relationship between two consecutive heartbeats, thus providing a visual representation of beat-to-beat variability over time. Despite the validation of this technique as means of analyzing HRV $[13,14]$, it remains to be applied clinically.

This study sets out to provide an initial proof-of-concept for a novel tool that utilizes HRV as a surrogate measure of autonomic function to provide real-time, accurate and noninvasive measurement of autonomic function that can be delivered at the point of care.

\section{Method}

\subsection{Ethical approval}

Ethical approval for data collection in this study was granted by the West Midlands Research Ethics Committee (NHS REC ID: 14/WM/0179, IRAS project ID: 156151). It was also conducted according to the UK Good Clinical Practice in Research (Research Governance Framework for Health and Social Care 2005) and Patients Protection Act 1998.

\subsection{Patients}

Anonymized continuous heart rate data recordings of 18 young and healthy (18-45 years) patients undergoing propofol anesthesia for arthroscopic surgery were used for analysis. Anesthesia was induced using a standardized protocol of propofol $(3.5 \mathrm{mg} / \mathrm{kg} \pm 1.3)$ followed by fentanyl $(1.6 \mathrm{mcg} / \mathrm{kg}$ $\pm 0.7)$, and a record was made of any further interventions, such as fluid challenges and vasoactive drug administration. All of the participants received positive pressure ventilation. The depth of anesthesia in patients was monitored using a BIS $^{\text {TM }}$ monitoring system with a target of 40-60.

Control data was extracted from the freely available online Fantasia dataset [15] from the Massachusetts institute of Technology (MIT), that includes anonymized heart rate data from healthy young volunteers (21-35 years) at rest. Fantasia is an open database created by the Massachusetts Institute of Technology in 1999 and was obtained on line. It contains the resting ECG recordings of 20 young (21-34 years old) and 20 elderly (68-85 years old) healthy subjects. We analysed data from 18 young subjects, to match the number of the patient dataset. ECG, respiratory rate and blood pressure recordings were made while the patients were lying supine and fairly still, and watching the Fantasia film.

\subsection{Data collection}

A 12-lead ECG was used to collect heart rate data in the Fantasia group, and a LiDCOrapid ${ }^{\mathrm{V} 2} \mathrm{CNAP}^{\circledR}$ Module (CNSystems Medizintechnik AG, Graz, Austria) was used to record cardiovascular parameter data in the patient group. The latter consists of a finger sensor that measures arterial diameter by means of infrared light, and an inflatable cuff over the proximal phalanx of the middle and index fingers

\section{Heart rate variability before (Stage 1) and after (Stage 2) anesthesia}

Stage 1

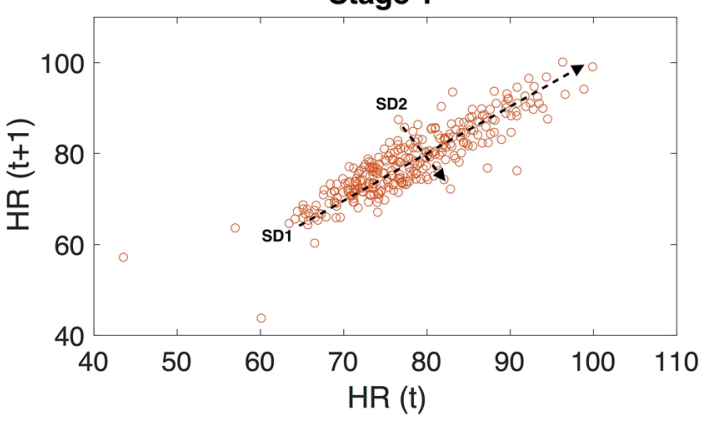

Fig. 1 Poincare plots before ('Stage 1'-left) and after ('Stage 2'right) anesthesia. Stage 1 anesthesia illustrates baseline data and it is defined as $5 \mathrm{~min}$ of recording prior to induction of anesthesia. Stage 2 anesthesia illustrates maintenance of anesthesia and it consists of 5 min taken during anesthesia. Changes in the plot shapes indicate
Stage 2

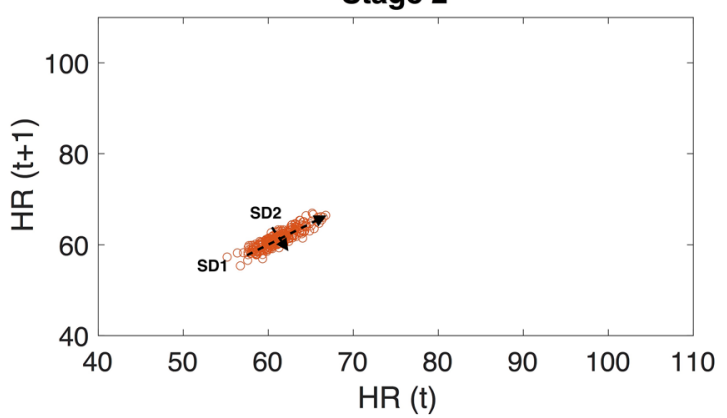

changes in parasympathetic (SD2) and sympathetic (SD1) tone. HR(t) indicates the $\mathrm{HR}$ at the first beat, whereas $\mathrm{HR}(\mathrm{t}+1)$ indicates the HR of the next heartbeat; each data point therefore represents the relationship between HR of two successive heart beats 
that monitors blood pressure. The CNAP system recorded heart rate and other cardiovascular parameters, with a sampling frequency of $100 \mathrm{~Hz}$; in the control dataset the ECG was sampled at $150 \mathrm{~Hz}$. Once the HR data was obtained, the HR time series of both patients and controls was resampled at $1 \mathrm{~Hz}$ for Poincaré plotting and analysis. Standardisation of the HR time series frequency was necessary to ensure homogeneity across datasets and to avoid artificially low HRV as a result of higher sampling frequencies (and vice versa). Standardisation is also necessary for Poincaré analysis, which relies on a uniform time difference between each time series point. In the patient group this was achieved by linear interpolation. In the control group this was achieved using the function 'tach' (WFDB toolbox for Matlab) [15, 16], which produces a uniformly sampled and smoothed instantaneous heart rate signal from heart-beat annotation files (the annotation files are available as part of the Fantasia dataset). Poincaré plots and SD1/2 measures were subsequently estimated from the HR data.

\subsection{Poincaré plot analysis}

Data analysis was performed in MatLab r2016b [17] (MATLAB R2016b. Natick, Massachusetts: ${ }^{\circ}$ The MathWorks Inc., 2016).

Poincaré plots are non-linear and geometrical representations of HRV. The plot is a cluster of points along the line $\mathrm{x}=\mathrm{y}$ (the line of identity), and every point on this line represents two heart beats of the exact equal rate, or successive identical RR intervals. Generally, any deviation above this line indicates an acceleration in heart rate form one beat to the next, and any deviation below it indicates a deceleration. The movement of points along the line represents long-term changes in heart rate. A wide, and long plot indicates high overall variability, which is indicative of a high level of autonomic tone, whereas narrow, bullet shaped plots indicate low HRV, and are typical of patients with a low level of autonomic function.

Poincaré plots can be analyzed both visually and geometrically. Visually, large, fan-shaped plots have been shown to indicate a prevalence of PSNS activity, whereas narrow and long, torpedo-like plots indicate a prevalence of SNS activity. Quantitatively, plots can be analyzed by fitting an ellipse to the distribution of points, and measuring the width of the distribution along (SD2) and perpendicularly to the identity line (SD1) [11, 12]. The 'width' of a Poincare plot (SD1) has been described as a direct measure of beat-to-beat variability, and therefore parasympathetic activity $[10,13]$, with a wider SD1 indicating higher parasympathetic tone. Similarly, Brennan et al. [14] linked sympathovagal balance to the 'length' of the Poincare plot (SD2) [18]. In this study, SD1 and SD2 are used to characterize HRV and, therefore autonomic function, in a young cohort of patients during surgery under propofol general anesthesia.

\subsection{Data extraction}

HRV was quantified when patients were awake and during anesthesia using Poincaré plots and their respective SD1 and SD2 measures based on the subjects' continuous heart rate readings. To this aim, two separate stages were extracted from each continuous recording of cardiovascular data:

(1) Stage 1 (before anesthesia), which consists of 150 $300 \mathrm{~s}$ window of HR data immediately preceding anesthetic induction;

(2) Stage 2 (during anesthesia), which consists of a 300-s window of HR data taken between the 15th and 30th min of surgery.

Care was taken to extract time segments that did not involve the administration of fluid challenges, vasoactive or ionotropic drugs for Stage 2 in patients undergoing anesthesia. In the control group, two 300-s intervals were extracted at the same time points for comparison: 0 to $5 \mathrm{~min}$, and 15-20 min. Values of heart rate and mean arterial pressure during Stage 1 and Stage 2 were also extracted for all patients.

HRV in each stage was analyzed using MatLab, using the Poincare SD1/SD2 estimation functions described in Piskorski et al. [12]. Data was filtered to remove noise and ectopic beats, whereby data exceeding a threshold $\pm 20 \%$ were excluded, as recommended by Karlsson et al. [19]. The SD1 and SD2 parameters at Stage 1 and 2 were then compared. Significance was tested using a Mann-Whitney-Wilcoxon test $(\mathrm{p}<0.05)$.

Furthermore, the real-time applicability of a Poincarébased tool of HRV dynamics was investigated. This analysis was performed on post-hoc data for proof-of-concept. A 'sliding window' model was created in order to sequentially analyse data and therefore to build a model for a tool that can be applied on continuous, live data. The Poincaré plot parameters SD1 and SD2 were estimated over $20 \mathrm{~s}$ windows, which were updated every $5 \mathrm{~s}$, therefore analysing data sequentially from start to end. Several possible window lengths for SD1/2 extraction were investigated, ranging from 5 to $120 \mathrm{~s}$, to obtain the highest resolution possible while preserving accuracy. Through these investigations it was found that a $20 \mathrm{~s}$ window provided the highest resolution with lowest levels of noise. Windows of less than $20 \mathrm{~s}$ resulted in graphs with large amounts of noise, because the measurement of SD1 and SD2 was, in such cases, derived from 5 consecutive beats or less. On the other hand, windows longer than $20 \mathrm{~s}$ did not result in reduction of noise but displayed data with less time resolution; the shortest window 
possible was therefore selected to provide the highest resolution with minimal noise.

\subsection{Outcome measure}

1. Estimation of SD1 (sympathetic tone-SNS) and SD2 (parasympathetic tone-PSNS) Poincaré parameters from HRV at baseline and during anesthesia.

2. Real-time modelling of HRV dynamics over the recording period, producing a model for autonomic monitoring in real-time.

\section{Results}

\subsection{Poincaré plot quantification: SD1 and SD2}

Quantification of Poincaré plots demonstrated visible changes in HRV (Fig. 1), which were mathematically quantifiable across the two stages. 'Stage 1' (baseline) SD1/2 were compared with 'Stage 2' values (during anesthesia). The comparison is shown in Fig. 2 and Table 1. Following anesthesia, both SD1 and SD2 values decreased, and these differences were significant $(p=0.019$ and $p=0.00027)$, thus indicating significant suppression of both SNS and PSNS activity. HRV observed in resting controls was unchanged between Stage 1 and Stage 2 (Fig. 3) (Table 1).

\subsection{Real-time monitoring: sliding window analysis}

SD1 and SD2 were measured sequentially in 20-s windows sliding by 5-s, and plotted throughout the surgery for each
Table 1 HRV quantification before ('Stage 1') and after ('Stage 2') anesthesia

\begin{tabular}{lcccc}
\hline & Mean & Median & IQR & $\mathrm{p}$ \\
\hline Patient SD1 & & & & \\
$\quad$ Stage 1 (baseline) & 3.53 & 3.24 & 1.86 & $0.019^{*}$ \\
$\quad$ Stage 2 (maintenance) & 1.86 & 0.96 & 1.33 & \\
Patient SD2 & & & & \\
$\quad$ Stage 1 (baseline) & 11.3 & 12.3 & 5.80 & $<0.001^{*}$ \\
Stage 2 (maintenance) & 4.35 & 3.68 & 4.02 & \\
Control SD1 & & & & \\
$\quad$ Stage 1 (0-5 min) & 1.51 & 1.40 & 0.69 & 0.950 \\
Stage 2 (15-20 min) & 1.46 & 1.46 & 0.70 & \\
Control SD2 & & & & \\
$\quad$ Stage 1 (0-5 min) & 5.28 & 5.29 & 1.83 & 0.393 \\
Stage 2 (15-20 min) & 5.22 & 4.41 & 2.97 & \\
\hline
\end{tabular}

Stage 1 anesthesia illustrates baseline data and it is defined as $5 \mathrm{~min}$ of recording prior to induction of anesthesia. Stage 2 anesthesia illustrates maintenance of anesthesia and it consists of 5 min taken during anesthesia. For the control data, Stage 1 consists of data from 0 to $5 \mathrm{~min}$ of the recording, and Stage 2 of data from 15 to $20 \mathrm{~min}$ of the recording. Significance was tested for using a Mann-Whitney-Wilcoxon $U$-test. Statistically significant difference are indicated with an asterisk

patient. Examples of graphs using varying sampling length are provided in Supplementary Figs. 1-5. This was used to graphically model HRV and, therefore, autonomic function dynamically and in real-time. There was a marked decrease in, and lower variation of, both SD1 and SD2 following administration of propofol to patients (Fig. 4). Similar trends in SD1 and SD2 were not observed in the control group (Fig. 5).

\section{SD1 and SD2 of patients before and after anesthesia}
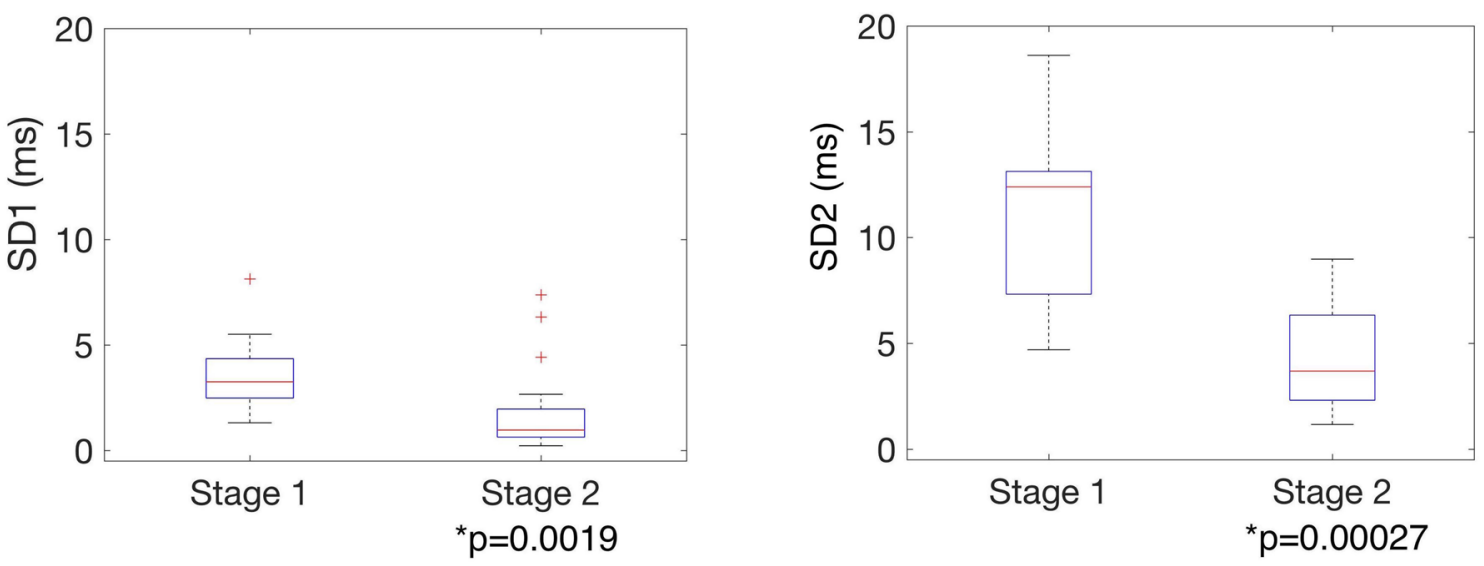

Fig. 2 Boxplots of average SD1 and SD2 before ('Stage 1'-left) and after (Stage 2'-right) anesthesia, indicating changes in parasympathetic and sympathetic tone between the two stages. $S D 1$ sympathetic function, $S D 2$ parasympathetic function 
Fig. 3 Boxplots of SD1 and SD2 from the resting controls taken at two time points during the recording, 'Stage 1' (left) and 'Stage 2' (right). SD1 sympathetic function, $S D 2$ parasympathetic function
SD1 and SD2 of control subjects at rest

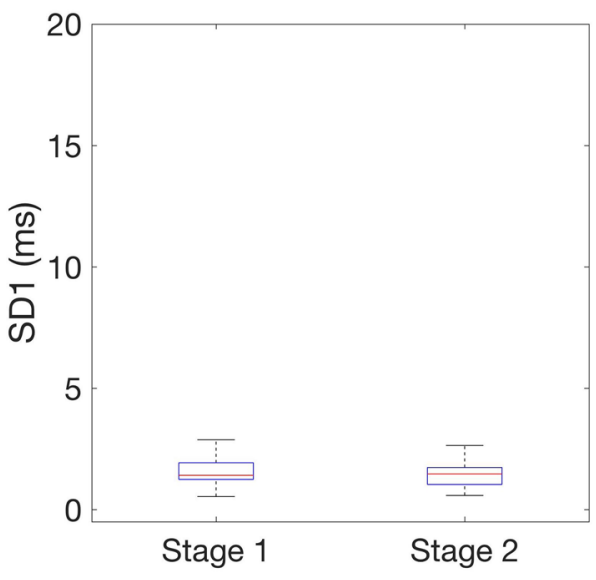

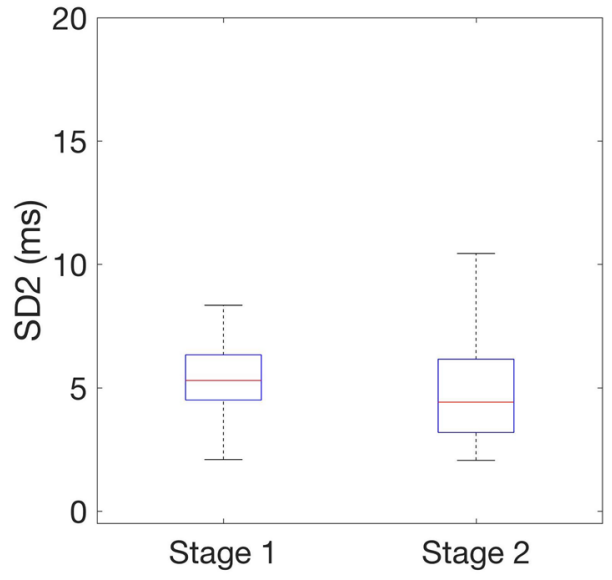

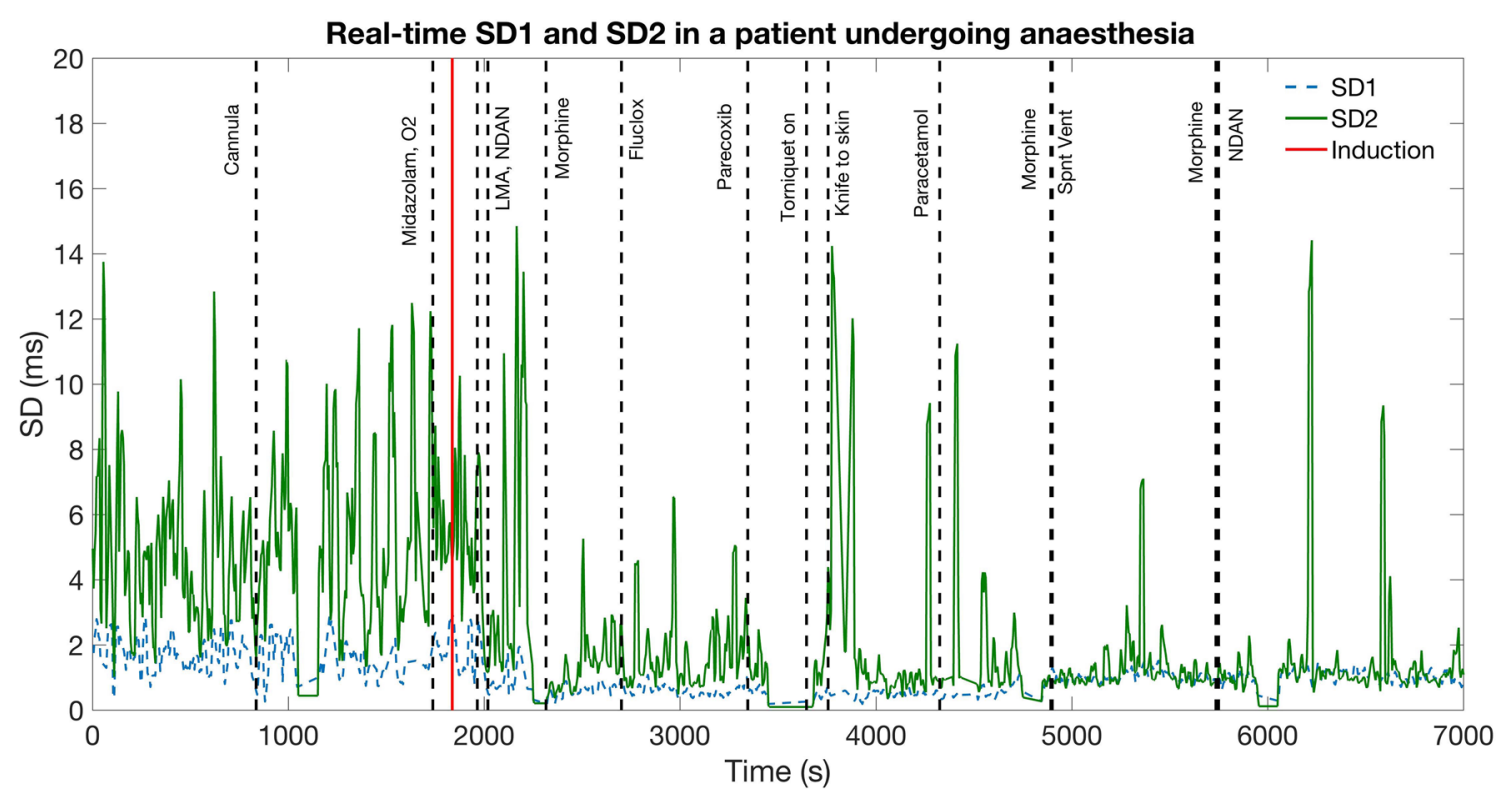

Fig. 4 Sliding window analysis of SD1 and SD2 of a patient undergoing anesthesia using a 20 -s window, indicating parasympathetic and sympathetic tone in real-time. SD1 sympathetic function, SD2 parasympathetic function. HRV is measured in SD, represenitng geo-

\section{Discussion}

This study demonstrates a potential use of Poincaré analysis as a means to non-invasively characterize autonomic function in real-time. The shape of the Poincaré plots of the patients markedly converged following anesthesia in terms of 'width' and 'length', thus reflecting a decrease in both sympathetic and parasympathetic tone. This was quantified mathematically and could be visually appreciated on the real-time sliding window graph. Results from this study therefore validate the use of Poincare plots for metrical measures of distribution of the data points along the identity line. LMA laryingeal mark airway, Fluclox flucloxacillin, NDAN ondansentron

HRV quantification. This has been shown in the literature: Kamen et.al. [9] decisively validated Poincaré analysis of HRV trends on patients whose autonomic function was modulated pharmacologically or orthostatically: the width of the Poincaré plots was reduced during SNS-stimulating head-up tilt and anticholinergic atropine administration, but was increased after scopolamine, a known parasympathetic stimulant. Other than Poincaré plots, further methods of HRV analysis include time-frequency analysis, which is also commonly used. This involves power spectral density (PSD) analysis of electrocardiogram (ECG) waveform data, which categorizes HRV values into high 


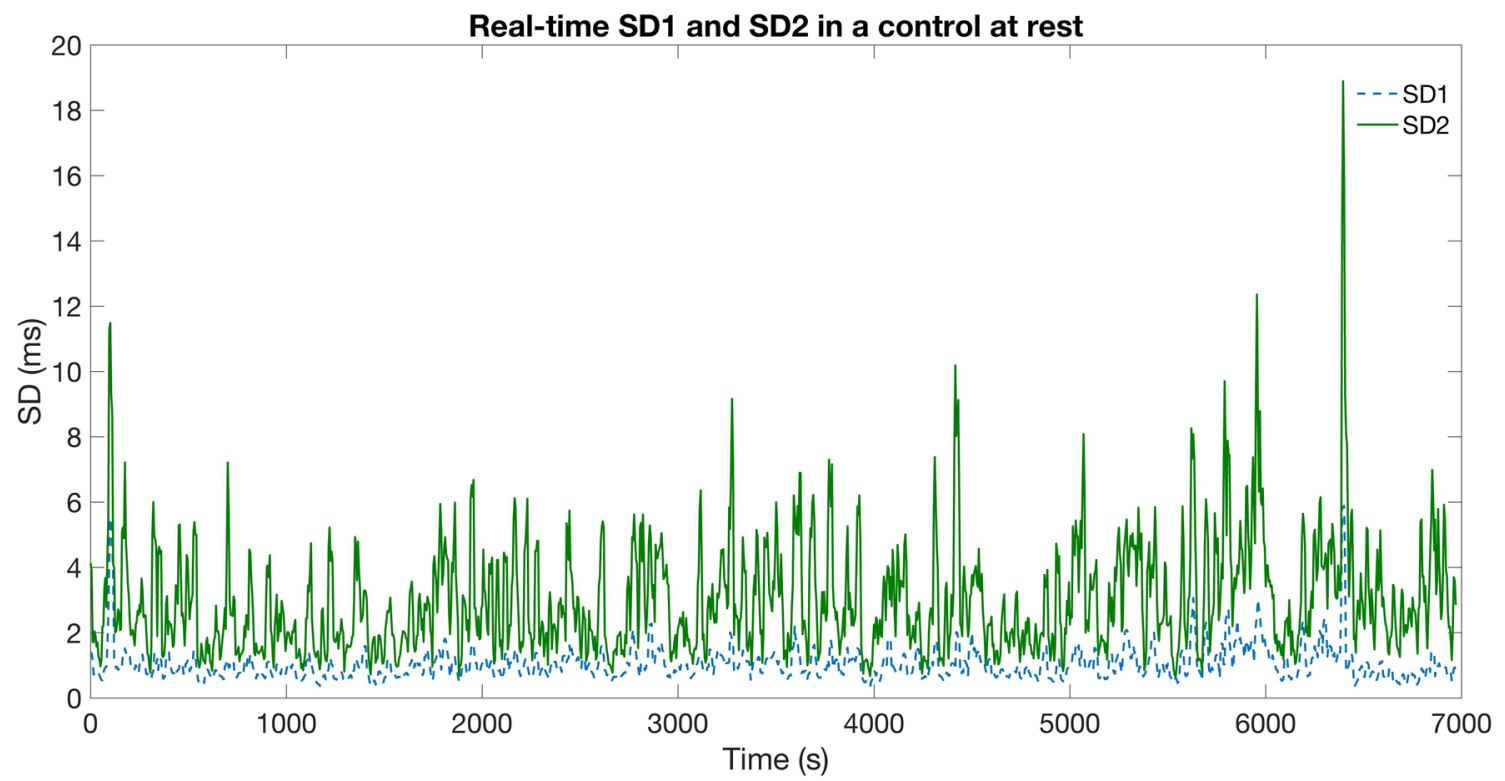

Fig. 5 Sliding window analysis of SD1/2 of a control subject during a resting period using a 20 -s window, indicating parasympathetic and sympathetic tone in real-time. $S D 1$ sympathetic function, $S D 2$ para-

frequency (HF), medium frequency (MF) and low frequency (LF) components with HF mainly reflecting PSNS activity, and LF the sympathovagal balance [20]. However, this is not a method that can be applied in real-time, and has been shown to be susceptible to high levels of respiratory noise [21]. Poincaré plots have been shown to be less affected by respiratory noise compared to other methods of heart rate variability analysis and correlate directly to PSD data [13, 14]: Poincaré width (SD2) reflects parasympathetic activation, and SD1 (length) reflects sympathetic antagonism to vagal tone. Furthermore, the SD1/SD2 ratio is analogous to the previously used spectral measure of LF/ HF ratio, indicating sympathovagal balance. Hsu et al. [18] performed a retrospective study on patients undergoing anesthesia to assess Poincaré plots as a means of assessing ANS modulation, and compared these to time-frequency analysis. Similarly to Brennan et al., a correlation was found between Poincaré and spectral measures, but Poincaré was deemed more accurate and easily obtainable. Hsu et al. reported that the autonomic suppression observed using HRV of patients undergoing anesthesia was dynamic and reflective of the known autonomic depressant effect of propofol in real-time [22, 23], which is in line with reported findings of the present study. Despite the numerous validations of its significance, Poincaré analysis of HRV has not yet been used as a real-time tool for clinical purposes. Our study presents a first proof-of-concept and could, therefore, contribute to the development of a means to routinely and conveniently measure autonomic function in patients at the point of care. This provides a scope for sympathetic function. HRV is measured in SD, represenitng geometrical measures of distribution of the data points along the identity line

personalization of anesthetic protocols and pre-surgical risk stratification. This is especially relevant in light of the increasing number of studies that have begun to investigate and prove the value of HRV in predicting complications of anesthesia and surgery [24, 25] (Fig. 6).

There are several limitations to consider. Firstly, the realtime analysis model was applied to data following, and not during, data collection. The designed tool was able to reflect changes in autonomic function in real-time graph by analysing data on a window-by-window basis. It therefore analysed the data sequentially as if in real-time, but the data used for the analysis had been pre-collected. Following positive results from this retrospective proof-of-concept data, further adjustment of the system will be needed to apply the tool to truly real-time data at the point of collection, rather than retrospectively. This study therefore provides a model, and not a finished product, for the analysis of data in real-time. However, it is important to note that the proposed HRV analysis model is transferable to live data without the need for developing a specialized module, via a direct interface between the patient monitor and a laptop device, where the HR data can be streamed and analysed directly, and an SD1/2-based index displayed on the laptop monitor. Software for such direct monitor-laptop interface already exist, e.g. VSCapture (open source software, https://sourceforge.net/projects/vscap ture/). Additional software may need to be implemented in order to calculate the SD1/2 indices, e.g., in Python (Python Software Foundation, https://www.python.org/). After an initial delay of 20-s (to buffer sufficient data as per the analysis presented in our work) the index can be updated every 


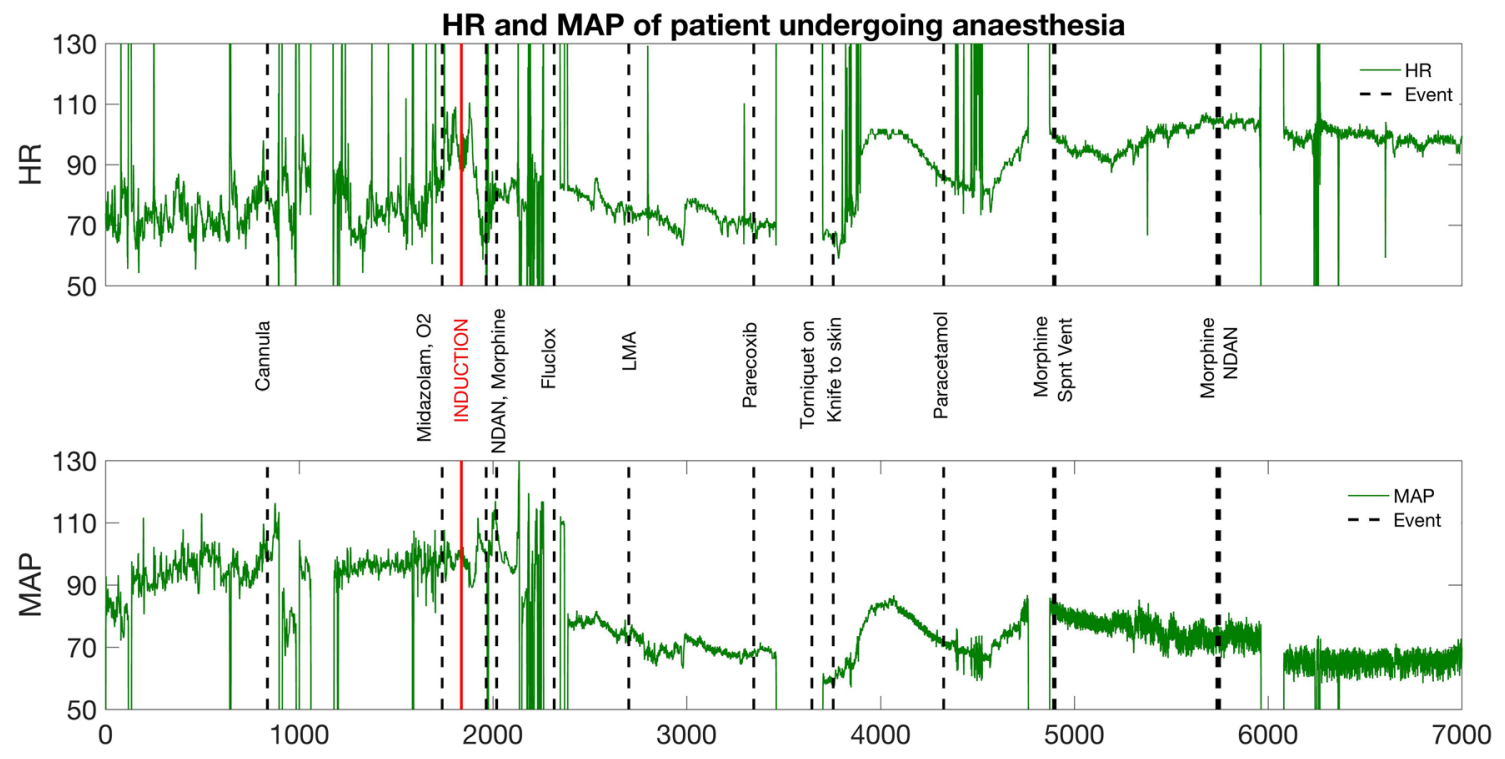

Fig. 6 Continuous monitoring data for heart rate and mean arterial pressure corresponding to subject undergoing anaesthesia whose HRV monitoring data is depicted in Fig. 4. LMA laryingeal mark airway, Fluclox flucloxacillin, NDAN ondansentron

second as per the model presented in this study. Secondly, the analysis tool that was constructed is based on continuous analysis of Poincaré plots derived from HR data. The plots constructed, therefore, reflect the relationship between two consecutive HR values. The HRV analysis was therefore based on pulse rate variability (PRV); this has been validated as a feasible and equivalent alternative to HRV calculation by RR-interval analysis for the analysis of HR data derived form pletysmography [26-28]. The rationale behind using HR estimated by the LidCO CNAP monitoring system and not using ECG-derived R-R intervals is mainly for reducing algorithmic complexity and improving accuracy. Estimation of R-R intervals from ECG requires additional analysis to estimate the exact locations of the QRS complexes and identification of the fiducial points, which is non-trivial in noisy or low amplitude ECGs. This is particularly important considering that recordings are performed in the noisy environment of the operating theatre where ECG sensors are susceptible to artifacts such as electrode movement (which introduces baseline wander in the measurements) and noise from the use of various surgical equipment. The HR data derived from the LiDCO CNAP finger sensor is less noisy and using this data directly allows a more flexible, cheaper and less complex 'plug-and-play' implementation. However, a limitation is that the SD1 and SD2 variables obtained from HR and RR intervals are not directly comparable. The conversion of HR data to RR interval data is relatively simple, but this would have added a further layer of complexity and, subsequently, potential error as the data would still reflect HR-derived RR intervals and not true RR intervals. It remains important to consider that differences in recording methods (12-lead ECG versus LidCO CNAP by finger plethysmography) may have given rise to intrinsic variation in the heart rate readings between controls and subjects. A further potential limitation of the study rests in the sampling frequencies of the data collection methods. The CNAP system records cardiovascular parameters with a sampling rate of $100 \mathrm{~Hz}$. Even though sampling frequencies greater than $250 \mathrm{~Hz}$ are generally recommended for HRV analysis (from ECG R-R intervals), Mahdiani and colleagues have shown that a sampling rate as low as $50 \mathrm{~Hz}$ could be used for measuring the ECG signal without compromising the accuracy of the calculated time domain HRV parameters [29]. Nevertheless, increasing the sampling frequency of the input data is a factor that warrants further investigation. Furthermore, before its clinical application, comparison to other previously validated tools available for assessment the ANS (e.g., state entropy, response entropy, Surgical Pleth Index [30] or Analgesia Nociception Index [31]), subtraction of the respiratory effect on HRV and further refining of the noise-reduction filter may also be warranted.

Consideration must be given to how patient morbidity may affect HRV. For example, conditions such as heart failure [32] and use of some medications [33] are likely to change HRV dynamics, and the impact of such variables investigated. During anesthesia itself, several factors may also affect HRV. Cardioactive agents (atropine, ephedrine), fluid boluses, and the effect of operative events on HRV must be considered, as these agents exert their effects by direct modulation of the autonomic nervous system, and are therefore likely to have a profound effect on HRV. The operating theatre is an inherently noisy environment, 
with artefacts arising from the procedure itself, such as intubation, knife to skin, diathermy etc., that are difficult and sometimes impossible (particularly in the case of diathermy) to remove. This is an issue even with commercially available systems, e.g., the BIS monitor stops displaying an index when diathermy is used. However, any measures derived from heart rate variability, such as SD1 and SD2, reflect the continuous modulation of the sinoatrial node and are, thus, bound to display some additional physiologically-related variation.

The ability to non-invasively and continuously measure HRV in real-time can be useful in clinical practice. The proposed tool has the potential to be employed in a continuous real-time monitoring system during anesthesia within the operating theatre, providing the anesthetist with direct, quantitative information about autonomic tone in real-time. Future development of this tool involves its trial on live data during collection, and the evaluation of its value as a preand in-surgical assessor and predictor of complications [34].

Our findings support the feasibility of Poincaré plot analysis for use in the development of a dynamic, non-invasive and real-time autonomic function characterization during anesthesia via the HRV. Such a tool would provide a faster, easier and more accessible ANS monitoring tool than the currently available methods of testing and, pending further development, has a wide scope for potential clinical applications.

Funding This study did not receive any specific grant from funding agencies in the public, commercial, or not-for-profit sectors.

\section{Compliance with ethical standards}

Conflict of interest None of the authors have any proprietary interests or conflicts of interest related to this submission.

Open Access This article is distributed under the terms of the Creative Commons Attribution 4.0 International License (http://creativeco mmons.org/licenses/by/4.0/), which permits unrestricted use, distribution, and reproduction in any medium, provided you give appropriate credit to the original author(s) and the source, provide a link to the Creative Commons license, and indicate if changes were made.

\section{References}

1. Cooper HE. Contribution of the respiratory rhythm to sinus arrhythmia in normal unanesthetized subjects during positivepressure mechanical hyperventilation. AJP Hear Circ Physiol. 2003;286(1):402H-11H. https://doi.org/10.1152/ajpheart.00504 2003 .

2. Zaza A, Lombardi F. Autonomic indexes based on the analysis of heart rate variability: a view from the sinus node. Cardiovasc Res. 2001;50(3):434-42. https://doi.org/10.1016/S0008 $-6363(01) 00240-1$.
3. Vita G, Princi P, Calabro R, Toscano A, Manna L, Messina C. Cardiovascular reflex tests. Assessment of age-adjusted normal range. J Neurol Sci. 1986;75(3):263-74. https://doi.org/10.1016/0022510X(86)90074-2.

4. Low PA, Opfer Gehrking TL, Proper CJ, Zimmerman I. The effect of aging on cardiac autonomic and postganglionic sudomotor function. Muscle Nerve. 1990;13(2):152-7. https://doi. org/10.1002/mus.880130212.

5. Tanaka M, Nishikawa T. Arterial baroreflex function in humans anaesthetized with sevoflurane. Br J Anaesth. 1999;82(3):3504. https://doi.org/10.1093/bja/82.3.350.

6. Widmark C, Olaison J, Reftel B, Jonsson LE, Lindecrantz K. Spectral analysis of heart rate variability during desflurane and isoflurane anaesthesia in patients undergoing arthroscopy. Acta Anaesthesiol Scand. 1998;42(2):204-10. https://doi. org/10.1111/j.1399-6576.1998.tb05110.x.

7. Ebert T, Muzi M. Propofol and autonomic reflex function in humans. Anesth Analg. 1994;78(2):369-75.

8. Hug CC Jr, McLeskey CH, Nahrwold ML, et al. Hemodynamic effects of propofol: data from over 25,000 patients. Anesth Analg. 1993;77(4 SUPPL.):21-9.

9. Trimmel K, Sacha J, Huikuri HV. Heart rate variability: clinical applications and interaction between HRV and heart rate. Lausanne: Frontiers Media SA; 2015. https://books.google.co. uk/books?id=VqEgCwAAQBAJ.

10. Kamen PW, Krum H, Tonkin AM. Poincaré plot of heart rate variability allows quantitative display of parasympathetic nervous activity in humans. Clin Sci. 1996;91(2):201-8. https://doi. org/10.1042/cs0910201.

11. Tulppo MP, Mäkikallio TH, Takala TE, Seppänen T, Huikuri HV. Quantitative beat-to-beat analysis of heart rate dynamics during exercise. Am J Physiol. 1996;271(1 Pt 2):H244-52. https ://doi.org/10.1016/0735-1097(94)90177-5.

12. Piskorski J, Guzik P. Geometry of the Poincaré plot of $R R$ intervals and its asymmetry in healthy adults. Physiol Meas. 2007;28(3):287-300. https://doi. org/10.1088/0967-3334/28/3/005.

13. Brennan M, Palaniswami M, Kamen P. Do existing measures of Poincaré plot geometry reflect nonlinear features of heart rate variability? IEEE Trans Biomed Eng. 2001;48(11):1342-7. https://doi.org/10.1109/10.959330.

14. Brennan M, Palaniswami M, Kamen P. Poincaré plot interpretation using a physiological model of HRV based on a network of oscillators. Am J Physiol. 2002;283(5):H1873-86. https://doi. org/10.1152/ajpheart.00405.2000.

15. Goldberger AL, Amaral LAN, Glass L, et al. PhysioBank, Physiotoolkit, and PhysioNet: components of a new research resource for complex physiologic signals. Circulation. 2000;101(23):e215-20.

16. Silva I, Moody G. An open-source toolbox for analysing and processing PhysioNet databases in MATLAB and Octave. J Open Res Softw. 2014;2(1):e27. https://doi.org/10.5334/jors.bi.

17. TM MATLAB r2016b Inc. MathWorks Inc; 2016. https://doi. org/10.1007/s10766-008-0082-5.

18. Hsu CH, Tsai MY, Huang GS, et al. Poincaré plot indexes of heart rate variability detect dynamic autonomic modulation during general anesthesia induction. Acta Anaesthesiol Taiwan. 2012;50(1):12-8. https://doi.org/10.1016/j.aat.2012.03.002.

19. Karlsson M, Hörnsten R, Rydberg A, Wiklund U. Automatic filtering of outliers in RR intervals before analysis of heart rate variability in Holter recordings: a comparison with carefully edited data. Biomed Eng Online. 2012;11(1):2. https://doi. org/10.1186/1475-925X-11-2.

20. El Beheiry H, Mak P. Effects of aging and propofol on the cardiovascular component of the autonomic nervous system. J 
Clin Anesth. 2013;25(8):637-43. https://doi.org/10.1016/j.jclin ane.2013.07.004.

21. Penttilä J, Helminen A, Jartti T, et al. Time domain, geometrical and frequency domain analysis of cardiac vagal outflow: effects of various respiratory patterns. Clin Physiol. 2001;21(3):36576. https://doi.org/10.1046/j.1365-2281.2001.00337.x.

22. Kanaya N, Hirata N, Kurosawa S, Nakayama M, Namiki A. Differential Effects of propofol and sevoflurane on heart rate variability. Anesthesiology. 2003;98(1):34-40. https://doi. org/10.1097/00000542-200301000-00009.

23. Nagashima Y, Furukawa Y, Hirose M, Chiba S. Cardiac effects of propofol and its interaction with autonomic nervous system in isolated, cross-circulated canine atria. J Anesth. 1999;13(1):34-9. https://doi.org/10.1007/s005400050019.

24. Bishop DG, Cairns C, Grobbelaar M, Rodseth RN. Heart rate variability as a predictor of hypotension following spinal for elective caesarean section: a prospective observational study. Anaesthesia. 2017;72(5):603-8. https://doi.org/10.1111/anae.13813.

25. Hanss R, Block D, Bauer M, et al. Use of heart rate variability analysis to determine the risk of cardiac ischaemia in high-risk patients undergoing general anaesthesia. Anaesthesia. 2008;63(1):1167-73. https://doi.org/10.111 1/j.1365-2044.2008.05602.x.

26. Jeyhani V, Mahdiani S, Peltokangas M, Vehkaoja A. Comparison of HRV parameters derived from photoplethysmography and electrocardiography signals. In: Proceedings of the Annual International Conference of the IEEE Engineering in Medicine and Biology Society, EMBS. 2015 November; 2015:5952-5955. https ://doi.org/10.1109/EMBC.2015.7319747.

27. Peng RC, Zhou XL, Lin WH, Zhang YT. Extraction of heart rate variability from smartphone photoplethysmograms. Comput Math Methods Med. 2015. https://doi.org/10.1155/2015/516826.
28. Yan BP, Chan CK, Li CK, et al. Resting and postexercise heart rate detection from fingertip and facial photoplethysmography using a smartphone camera: a validation study. JMIR mHealth uHealth. 2017;5(3):e33. https://doi.org/10.2196/mhealth.7275.

29. Mahdiani S, Jeyhani V, Peltokangas M, Vehkaoja A. Is $50 \mathrm{~Hz}$ high enough ECG sampling frequency for accurate HRV analysis? In: Proceedings of the Annual International Conference of the IEEE Engineering in Medicine and Biology Society, EMBS. 2015 November; 2015:5948-5951. https://doi.org/10.1109/ EMBC.2015.7319746.

30. Ledowski T, Burke J, Hruby J. Surgical pleth index: prediction of postoperative pain and influence of arousal. $\mathrm{Br} \mathbf{J}$ Anaesth. 2016;117(3):371-4. https://doi.org/10.1093/bja/aew226.

31. Ledowski T, Tiong WS, Lee C, Wong B, Fiori T, Parker N. Analgesia nociception index: evaluation as a new parameter for acute postoperative pain. BJA. 2013;111(4):627-9.

32. Brouwer J, Van Veldhuisen DJ, In't AJM et al. Prognostic value of heart rate variability during long-term follow-up in patients with mild to moderate heart failure. J Am Coll Cardiol. 1996;28(5):1183-9.

33. Michaloudis D, Kochiadakis G, Georgopoulou G et al. The influence of premedication on heart rate variability. Anaesthesia. 1998;53(5):446-53. https://doi.org/10.104 6/j.1365-2044.1998.00323.x.

34. Hanss R, Renner J, Ilies C et al. Does heart rate variability predict hypotension and bradycardia after induction of general anaesthesia in high risk cardiovascular patients? Anaesthesia. 2008;63:129135. https://doi.org/10.1111/j.1365-2044.2007.05321.x. 